\title{
General Linear Scaling Implementation of Polarizable Embedding Schemes
}

\author{
Filippo Lipparini* \\ Dipartimento di Chimica e Chimica Industriale, Università di Pisa, Via G. Moruzzi 13, \\ 56124 Pisa, Italy \\ E-mail: filippo.lipparini@unipi.it
}

\begin{abstract}
A general framework to treat polarizable embedding schemes in the context of $\mathrm{QM} / \mathrm{MM}$ calculations is presented. Such a framework is completely general, as it allows in principle one to treat any electrostatic distribution and polarization model with minimal modifications, and achieves linear scaling in computational cost and memory requirements. The performances and scaling of the new implementation are demonstrated with benchmark calculations on water clusters including up to 1146240 atoms.
\end{abstract}

Polarizable Embedding (PE) QM/MM is, nowadays, still not a standard technique. Despite a large number of different models and implementations ${ }^{1-8}$ available in various codes - often, in development versions of such codes - PE QM/MM is not as well established as electrostatic embedding (EE) QM/MM. The majority of the implementations are the result of active research and developments, and are tailored to a specific force field or polarization model. In fact, polarization can be introduced in a classical description by means of fluctuating charges (FQ), Drude oscillators (DO) or induced point dipoles (IPD). Furthermore, polarizable degrees of freedom can be accompanied by a permanent electrostatic distribution 
(ED) in the form of point charges or higher order multipoles. Due to these diversities, there is no common framework for PE QM/MM. Besides the technical difficulties associated with $\mathrm{PE} \mathrm{QM} / \mathrm{MM}$, the diffusion of such a technique is also limited due to its computational cost, which is considerably higher than in EE. ${ }^{9}$ In fact, PE models require one to determine the polarization degrees of freedom, which requires to solve a set of polarization equations. This is further aggravated from the fact that such equations need to be solved self-consistently with the QM equations, as mutual polarization between the classical and QM densities is included in the model. As an example, a PE QM/MM calculation where the QM core is described with Density Functional Theory (DFT) requires one to solve the polarization equations at each self-consistent field (SCF) cycle. ${ }^{1,6}$ The issue of computational cost becomes particularly relevant when many computation are to be performed in order to achieve a good statistical sampling of the environment ${ }^{10}$ or, even more, when ab-initio polarizable QM/MM molecular dynamics simulations are pursued ${ }^{11,12}$

Despite the many physical differences among the various polarization models, it is possible to find several formal similarities. Let us analyze the main step of a PE QM/MM calculation, choosing DFT as a QM level of theory in order not to complicate the discussion. Independent of the force field used, such a calculation requires one to perform the following steps:

1. Compute the interaction energy of the ED, if any.

2. Compute the inducing potential or electric field generated by the ED, if any

3. At each SCF cycle:

- Compute the inducing potential or electric field generated by the QM charge density

- Solve the polarization equations and compute the polarization energy

- Compute the PE contributions to the QM Hamiltonian

From this simple list of operations, it should emerge that from a mathematical point of 
view the PE-specific operations to be performed can be classified into two different groups, i.e., computing MM electrostatic properties (potential, field, ...) at the MM sites and solving MM polarization equations. A further look to the latter step makes this distinction inessential when one solves the polarization equations iteratively. In fact, the matrix/vector products (MVPs) required to solve iteratively the polarization equations can be, mutatis mutandis, associated to the evaluation of an electrostatic property where the polarization degrees of freedom are the sources. ${ }^{9}$ For IPDs, the MVP corresponds to the evaluation of the electrostatic field generated by the induced dipoles, ${ }^{9}$ for FQs to the potential of the fluctuating charges ${ }^{6}$ and for Drude oscillators to the electric field of the charges. ${ }^{7}$ As a consequence, all the operations involved in performing a PE QM/MM calculations can be seen as the evaluation of modified electrostatic quantities of multipolar distributions with maximum angular momentum depending on the nature of the polarization model.

In order to illustrate this idea and to define in detail in what sense the electrostatic quantities are modified in the most general way, let us consider a PE model based on IPD and with a ED made by Cartesian multipole moments up to the quadrupole. This is the case of the polarizable embedding model $^{2}$ and, with slight differences in the IPD formulation, of the QM/AMOEBA model. ${ }^{3}$ Other IPD models, as well as Drude and FQ based ones, can be seen as a variation of this example. Let $\mathbf{M}=\left\{M_{i}^{L}\right\}$ be the distribution of Cartesian multipoles of angular momentum $L=0,1,2$ placed at sites $i=1,2, \ldots, N$ and let $\mathbf{r}_{i}$ be the position of the $i-t h$ site. For the sake of notation simplicity, the Cartesian indices of the various tensors are not reported, and implicit sums are always assumed.

The first ingredient for the example is the interaction energy of a multipolar distribution, which is given by

$$
\mathcal{E}=\frac{1}{2} \sum_{i} \sum_{L_{1}} \sum_{j \neq i} \sum_{L_{2}} M_{i}^{L_{1}} T_{i j}^{L_{1} L_{2}} M_{j}^{L_{2}}
$$

where

$$
\left[T_{i j}^{L_{1} L_{2}}\right]_{\alpha^{\prime} \beta^{\prime} \ldots}^{\alpha \beta \ldots}=\frac{\partial^{L_{1}}}{\partial r_{i}^{\alpha} \partial r_{i}^{\beta} \ldots \partial r_{j}^{\alpha^{\prime}} \partial r_{j}^{\beta^{\prime}} \ldots\left|\mathbf{r}_{i}-\mathbf{r}_{j}\right|}
$$


Equation 1 can be interpreted as a two-steps process to compute the energy. Let

$$
\Phi_{i}^{L_{1}}=(-1)^{L_{1}} \sum_{j \neq i} \sum_{L_{2}} T_{i j}^{L_{1} L_{2}} M_{j}^{L_{2}}
$$

be the electrostatic property of order $L_{1}$ (i.e., potential, field, field gradient,...) generated by the multipolar distribution M. Eq. 1 can be equivalently rewritten as

$$
\mathcal{E}=\frac{1}{2} \sum_{i} \sum_{L_{1}}(-1)^{L_{1}} M_{i}^{L_{1}} \Phi_{i}^{L_{1}}
$$

that is, the energy of the distribution can be obtained by contracting the multipoles at site $i$ with the corresponding electrostatic property generated by every other multipole at the same site. The $(-1)^{L_{1}}$ factor that has been introduced in eqs. 3 and 4 is purely cosmetic, and serves a consistency purpose, as it allows one to follow the normal convention of defining the electric field as minus the gradient of the potential and the interaction energy of a set of multipoles as charge times potential, minus dipole times field and so on.

The IPD polarization equations are easily derived from variational considerations ${ }^{13}$ and are given by

$$
\alpha_{i}^{-1} \mu_{i}+\sum_{j \neq i} T_{i j}^{11} \mu_{j}=E_{i}(M, \mathbf{P}),
$$

where at the right-hand side we denote explicitly the dependence of the electric field on both the static multipoles and the QM density. The QM field can be computed using standard one-electron integrals machinery, ${ }^{3}$ while its MM counterpart can be assemble by using again eq. 3:

$$
E_{i}(M)=-\sum_{j \neq i} \sum_{L_{2}} T_{i j}^{1 L_{2}} M_{j}^{L_{2}}
$$

Eqs. 3 and 4 provide all the tools needed to implement a PE method. However, one has to be careful that such equations need to be modified in order to take into account two distinct, but equivalently important, aspects: 
- In force fields, interactions among topologically close atoms (i.e., 1-2, 1-3, ... neighbors) are already included in the so-called bonded interactions (stretching, bending, torsions, ...). The electrostatic interaction among multipoles placed on close atoms are therefore scaled or removed. ${ }^{14,15}$ From a formal point of view, this requires one to modify eq. 1 (or, equivalently, eq. 3) introducing for each couple of sites a scaling factor $s_{i j}$ that depends on the connectivity:

$$
\mathcal{E}=\frac{1}{2} \sum_{i} \sum_{L_{1}} \sum_{j \neq i} \sum_{L_{2}} s_{i j} M_{i}^{L_{1}} T_{i j}^{L_{1} L_{2}} M_{j}^{L_{2}}
$$

- In polarizable force fields, particular care is required when computing the polarization degrees of freedom. As the Coulomb kernel and its derivatives (eq. 2) diverge when two sites get close, it is possible to incur the so-called polarization catastrophe, i.e., unphysically large or even diverging polarization. To avoid this problem, the Coulomb kernel is usually damped when computing polarization interactions. ${ }^{14,15}$ This is done by replacing the bare Coulomb kernel with a dressed one, ${ }^{16}$ i.e., by defining

$$
\mathcal{T}_{i j}^{00}=\frac{\lambda_{1}\left(\left|\mathbf{r}_{i}-\mathbf{r}_{j}\right|\right)}{\left|\mathbf{r}_{i}-\mathbf{r}_{j}\right|}
$$

In figure 1 (left), a few possible choices for the damping function $\lambda_{1}\left(\left|\mathbf{r}_{i}-\mathbf{r}_{j}\right|\right)$ are reported and compared with the bare Coulomb kernel. Many possible choices exist, but they all share two main features. First, the damped kernel goes to a finite limit when $\mathbf{r}_{i} \rightarrow \mathbf{r}_{j}$. Second, they converge very quickly to the bare Coulomb Kernel when the distance increases. In particular, it can be seen from fig. 1 that for distances of a few Bohrs the damped and bare kernels become indistinguishable. To summarize, any polarizable force field which includes ED and polarization can be implemented using a limited number of common ingredients, namely, eqs. 3 and 4, connectivity information and scaling rules, and a damping function. Both eq. 3 and its modified versions can be implemented easily and in a general fashion, as 


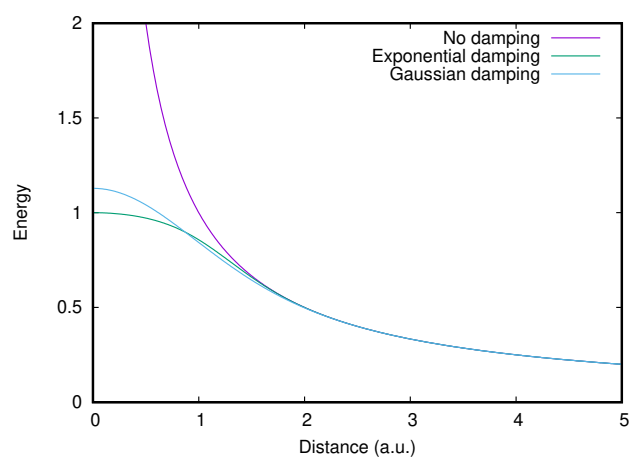

Figure 1: Interaction energy of two unit charges with bare and damped Coulomb kernels, for two possible choices of the damping function. The Exponential choice dresses the point charges with an exponential function, the Gaussian one with a Gaussian function. All data are in atomic units.)

recursions exist that allow one to derive and implement them for arbitrary order multipolar expansions.

Achieving Linear Scaling in Computational Cost Recognizing the common framework presented in this communication has a second, important advantage. From a computational point of view, all the operations required for the implementation of a PE QM/MM scheme consist in either the evaluation of eq. 3 or eq. 4 , the former possibly modified to account for scaling or damping. Eq. 4 can be evaluated with $\mathcal{O}(N)$ operations, where $N$ is the number of MM atoms, and does not represent a computational bottleneck. On the contrary, evaluating eq. 3 requires one to perform $\mathcal{O}\left(N^{2}\right)$ operations. Given that such an operation needs to be performed several times, as it is also required in order to compute a step during the iterative solution of the polarization equations, it represents the expensive step in a PE QM/MM computation, as well as in a EE QM/MM one. An efficient implementation of $\mathrm{PE}(\mathrm{EE}) \mathrm{QM} / \mathrm{MM}$ can therefore only be achieved if a fast, efficient and general strategy is employed for the calculation of the electrostatic properties of a multipolar distribution. On the other hand, thanks to the generality of the formalism, this is also the only computational problem that needs to be addressed.

A standard fast summation technique to evaluate eq. 3 at $\mathcal{O}(N)$ cost is provided by 
the Fast Multipole Method ${ }^{17}$ (FMM). The FMM was originally formulated for point charges distributions, but has been extended to dipolar ones and even to continuous charge distributions. ${ }^{18}$ However, arbitrary order multipolar distributions, the presence of scaling rules and the use of a damped kernel require some further work. In order to illustrate the main ideas

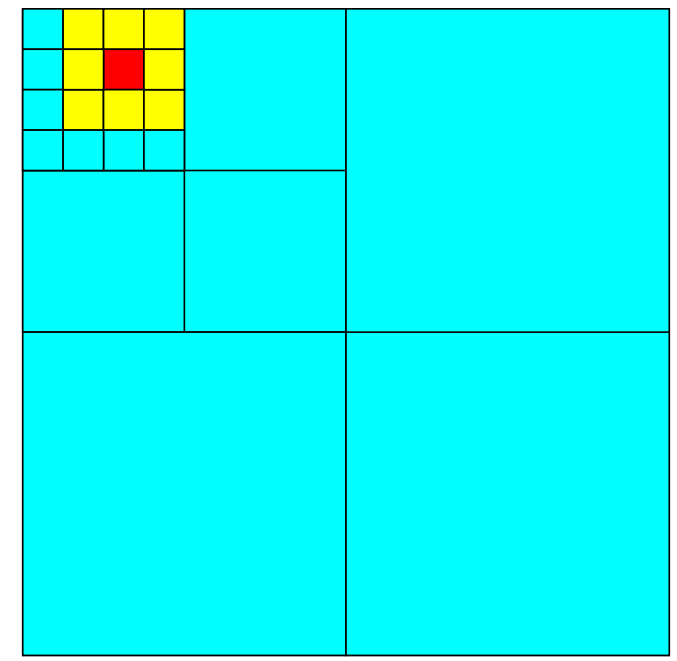

Figure 2: A 2D pictorial representation of the boxification if FMM. The figure depicts three boxification levels. In the upper left part, the finest level is represented. Particles inside the red box interact among themselves and with particles inside the yellow boxes within the near field regime. Interaction with all other boxes (cyan) is described in the far field regime.

underlying the FMM (an excellent and not too technical review on the FMM can be found in ref. 19), let us consider a distribution of point charges. The FMM procedure requires one to encapsulate such a distribution into a cubic box of side $a_{0}$, which is then divided into 8 boxes of side $a_{1}=a_{0} / 2$ and so on recursively, until the smallest box only contains a small amount of charges or has reached a minimum side length set by the user. A two-dimensional sketch of the boxification can be found in figure 2. The interaction among the charges is computed differently depending on whether two sites belong to the same or to a neighboring box, or to a well separated one. In the former case, usually called near field, the interaction is computed using eq. 3. In the latter case ( far field), the interaction is computed as follows. A multipolar distribution is assembled in each box (particle to multipole step, P2M) at the finest level using the charges that belong to the box itself. Distributions from smaller boxes are merged in order to evaluate the ones in parent boxes (multipole to multipole step, M2M) 
until the coarsest level is reached. Then, the multipolar distributions are converted into local expansions (multipole to local, M2L), which are in turn translated and merged down the box tree until a local expansion is available in each box at the finest level (local to local, L2L). Finally, the local expansions are used to evaluate the potential and thus the coulomb interaction energy with the charges in the smallest boxes (local to particle, L2P).

Among all the steps required for the evaluation of the far field, only the P2M and L2P ones are associated with a computational cost that depends on the number of particles: as these steps are linear, the overall far field evaluation scales as $\mathcal{O}(N)$. The near field evaluation, which is limited to a small number of close particles, also scales linearly with the size of the system. Therefore, the FMM makes the evaluation of the interaction energy among charged particles possible with $\mathcal{O}(N)$ operations. More details can be found in the Supporting Information.

The use of a FMM library for PE QM/MM requires some generalizations. First, standard FMM libraries do not support damped interactions. However, the damping functions decay very quickly, as it can be seen from fig. 1 . Therefore, by choosing a minimum box side larger than the distance at which the difference between the bare and damped kernels becomes negligible, damping can be handled exclusively in the near field, which can be easily modified. This is in principle very delicate, as the efficiency and accuracy of the overall FMM procedure depends on the box side. Fortunately, the decay of the damping functions is usually fast enough that box sizes that are optimal for performances are also adequate to treat damping.

Second, the sources can be arbitrary order multipoles. This is not an issue for the evaluation of the near field, as eq. 3 can be used, possibly in its damped version, without further modification. The evaluation of the far field, on the other hand, requires modifications to both the P2M and L2P steps. The former is easily achieved by using the same machinery used for the M2M step. The multipolar distributions on the MM atoms can be translated to the center of the box where they belong using a M2M transformation and then summed together. The L2P step needs to be modified because, in order to deal with higher order 
multipoles, one needs to compute higher order derivatives of the potential (i.e., field, field gradient, ...). As the potential is evaluated by contracting the local expansion with regular solid spherical harmonics, this is easily achieved by recursion. ${ }^{20}$

Finally, the connectivity dependent scaling of the interactions can be dealt with after the computation, by simply removing or rescaling the unwanted interactions. This means that one evaluates the potential as

$$
\Phi_{i}^{L_{1}}=(-1)^{L_{1}} \sum_{j \neq i} \sum_{L_{2}} \mathcal{T}_{i j}^{L_{1} L_{2}} M_{j}^{L_{2}}+(-1)^{L_{1}} \sum_{j \neq i} \sum_{L_{2}}\left(s_{i j}-1\right) \mathcal{T}_{i j}^{L_{1} L_{2}} M_{j}^{L_{2}}
$$

The first term of eq. 9 is evaluated with the modified FMM procedure previously illustrated, while the second term, that corrects for the scaling, is added afterwards. It is important to note that the $s_{i j}$ factors are different from one only for a small set of atoms that are either directly bonded or a few bonds away from each other. By constructing 1-2, 1-3, .. neighbor lists, it is thus possible to evaluate the correction at a cost that scales linearly with the size of the system.

Implementation and Benchmark Calculations The general framework described in this work has been implemented in a locally modified development version of the Gaussian suite of programs. ${ }^{21}$ The new implementation builds on the internal FMM library implemented in Gaussian ${ }^{22}$ and on a pilot implementation by the author and coworkers. ${ }^{23}$ The previous implementation, which could only treat point charges distributions and induced dipoles, with a specific set of exclusion rules and damping function, has been completely rewritten and generalized to treat arbitrary combinations of exclusion rules, damping functions and order of the multipolar distribution. The machinery has also been extended to the evaluation of the forces. Currently, functionalities are implemented to manage IPD, ED up to the quadrupole, exponential and polynomial ${ }^{14}$ damping functions and general exclusion rules based on connectivity information. Nevertheless, generalizations to other models, damping functions, higher order EDs or even higher order derivatives can be achieved with 
small and trivial modifications.

In order to demonstrate the capabilities of the new implementation, PE QM/MM benchmark calculations are performed using either the MMPol model (point charges and polarizable point dipoles using the parameters of the AMBER ff02 polarizable force field by Wang et al. ${ }^{14}$ ) or the AMOEBA force field. ${ }^{15}$ To demonstrate the linear scaling and overall efficiency of the implementation, the calculations are performed on spherical water clusters of increasing size. In MMPol, water molecules are described with three point charges and a single isotropic polarizability, while in AMOEBA each atom is endowed with a static charge, dipole and quadrupole and with an isotropic polarizability. It is also to be noted that the AMOEBA polarization energy requires one to compute two sets of induced dipoles, as a consequence of different exclusion rules being used fo the MM inducing field and for the calculation of the MM polarization energy. Globular systems are known to be the worst case scenario for FMM, as they maximize the number of relevant interactions. For this reason, they represent a challenging, but significant benchmark for the new implementation. For each cluster, one single water molecule is used as the QM core, while all the others belong to the PE region. All the calculations have been performed on a single computer node equipped with two Xeon E5-2620 v2 2.10GHz and 64 GB of memory. We report in fig. 3 the timings of the operations needed in order to perform a PE QM/MM calculation, namely, the calculation of the ED electrostatic interaction and of its inducing field(s) at the polarizable sites, the solution to the polarization equations (two linear systems for AMOEBA) at the first SCF cycle, and the computation of the PE contributions to the forces. More details are given in the supporting information. The linear scaling in computational cost is apparent from the figures for all computations. As expected, the AMOEBA calculations are considerably more expensive than their MMPol counterpart. This is due both to the presence of two different sets of induced dipoles and of higher order quadrupoles, and to the fact that the number of polarizable sites is three times its counterpart in the MMPol calculation, as all atoms bear a polarizability. Nevertheless, the presence of a polarizable embedding introduces a modest 

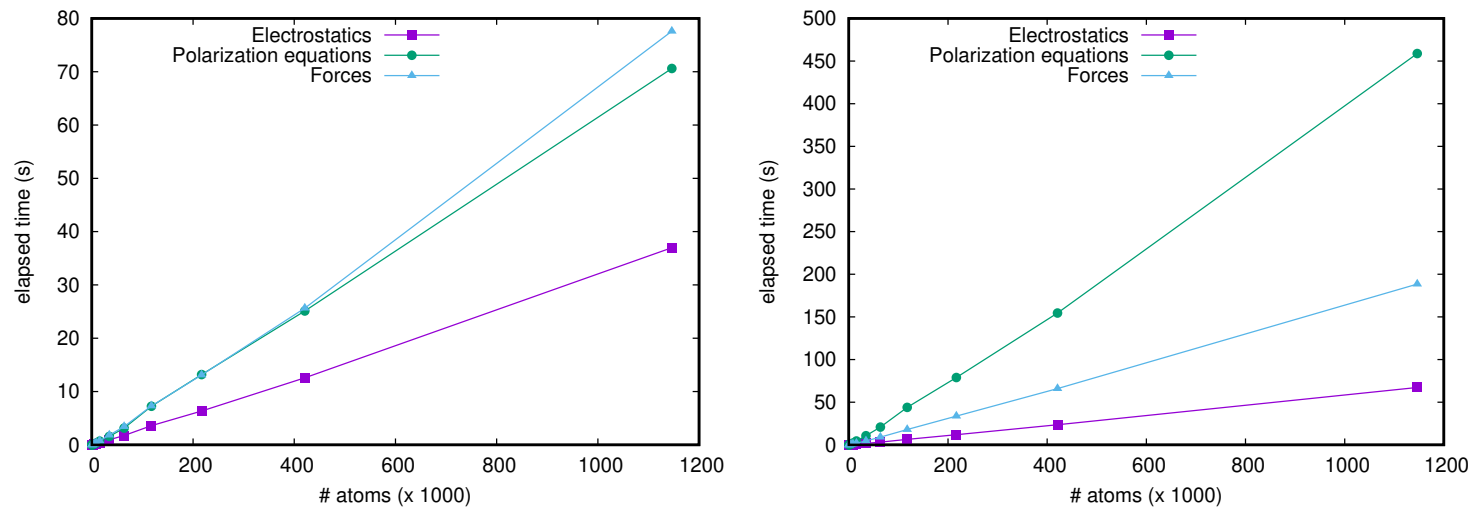

Figure 3: Elapsed time (in seconds) as a function of the system size (in thousands of atoms) for the evaluation of the permanent electrostatic distribution interaction energy and of the MM inducing field(s) (Electrostatics), for the solution to the polarization equations and for the evaluation of the PE contributions to the forces for MMPol (left panel) and AMOEBA (right panel) PE QM/MM calculations.

computational overhead even for the largest system tested, which is thus easily treatable on a routine basis even using modest computational hardware such as the one used for this tests.

In conclusion, it is possible to define a common framework for the evaluation of the electrostatic interactions, the solution of the polarization equations, and the calculation of the polarization energy which can be used to treat static multipolar distributions of arbitrary order in conjunction to polarization, described with any model. This is done by recognizing that the elementary operations that define any PE calculation can be seen as either the evaluation of an electrostatic property, or its contraction with a distribution. The calculation can be performed efficiently and at a cost that scales linearly with the size of the polarizable environment. Such a cost is overall modest even for extremely large embeddings, such as the ones employed for the tests presented in this work. This work opens thus the way not only to a more standardized implementation of PE QM/MM schemes, but also to their routine application to large and complex chemical problems on a routine basis. This is in line with the recent efforts ${ }^{12}$ of the author and coworkers to create an infrastructure suitable for polarizable QM/MM ab-initio molecular dynamics simulations based on the coupling of the Gaussian and Tinker ${ }^{24} /$ Tinker $\mathrm{HP}^{25}$ suites of programs. The current implementation 
allows in fact the treatment of very large embeddings with limited overhead with respect to the cost of treating the QM portion of the system, enabling thus applications on complex biological systems.

\section{Supporting Information}

A complete description of multipolar interactions and explicit formulae for the energy and forces of charges, dipoles and quadrupoles, a more complete description of the FMM and computational details of the benchmark calculations are available in the supporting information.

\section{Acknowledgments}

The author would like to thank prof. Benedetta Mennucci and prof. Jean-Philip Piquemal for their useful comments on the manuscript. Discussion with Dr. Giovanni Scalmani and Dr. Michael J. Frisch on the FMM is also greatly appreciated. Financial support from the PRA_2018_36 project is gratefully acknowledged.

\section{References}

(1) Curutchet, C.; Muñoz-Losa, A.; Monti, S.; Kongsted, J.; Scholes, G. D.; Mennucci, B. Electronic Energy Transfer in Condensed Phase Studied by a Polarizable QM/MM Model. J. Chem. Theory Comput. 2009, 5, 1838-1848.

(2) Olsen, J. M. H.; Kongsted, J. In Chapter 3 - Molecular Properties through Polarizable Embedding; Sabin, J. R., Brändas, E., Eds.; Adv. Quantum Chem.; Academic Press, 2011; Vol. 61; pp $107-143$.

(3) Loco, D.; Polack, É.; Caprasecca, S.; Lagardère, L.; Lipparini, F.; Piquemal, J.-P.; 
Mennucci, B. A QM/MM Approach Using the AMOEBA Polarizable Embedding: From Ground State Energies to Electronic Excitations. J. Chem. Theory Comput. 2016, 12, $3654-3661$.

(4) Dziedzic, J.; Mao, Y.; Shao, Y.; Ponder, J.; Head-Gordon, T.; Head-Gordon, M.; Skylaris, C.-K. TINKTEP: A fully self-consistent, mutually polarizable QM/MM approach based on the AMOEBA force field. J. Chem. Phys. 2016, 145, 124106.

(5) Dziedzic, J.; Head-Gordon, T.; Head-Gordon, M.; Skylaris, C.-K. Mutually polarizable QM/MM model with in situ optimized localized basis functions. J. Chem. Theory Comput. 2019, 150, 074103.

(6) Lipparini, F.; Cappelli, C.; Barone, V. Linear response theory and electronic transition energies for a fully polarizable QM/Classical Hamiltonian. J. Chem. Theory Comput. 2012, 8, 4153-4165.

(7) Boulanger, E.; Thiel, W. Solvent Boundary Potentials for Hybrid QM/MM Computations Using Classical Drude Oscillators: A Fully Polarizable Model. J. Chem. Theory Comput. 2012, 8, 4527-4538.

(8) Lev, B.; Zhang, R.; de la Lande, A.; Salahub, D.; Noskov, S. Y. The QM-MM interface for CHARMM-deMon. J. Comput. Chem. 2010, 31, 1015-1023.

(9) Lipparini, F.; Lagardère, L.; Stamm, B.; Cancès, E.; Schnieders, M.; Ren, P.; Maday, Y.; Piquemal, J.-P. Scalable Evaluation of Polarization Energy and Associated Forces in Polarizable Molecular Dynamics: I. Toward Massively Parallel Direct Space Computations. J. Chem. Theory Comput. 2014, 10, 1638-1651.

(10) Lipparini, F.; Egidi, F.; Cappelli, C.; Barone, V. The Optical Rotation of Methyloxirane in Aqueous Solution: A Never Ending Story? J. Chem. Theory Comput. 2013, 9, 18801884. 
(11) Loco, D.; Lagardère, L.; Caprasecca, S.; Lipparini, F.; Mennucci, B.; Piquemal, J.P. Hybrid QM/MM Molecular Dynamics with AMOEBA Polarizable Embedding. J. Chem. Theory Comput. 2017, 13, 4025-4033.

(12) Loco, D.; Lagardère, L.; Cisneros, G. A.; Scalmani, G.; Frisch, M.; Lipparini, F.; Mennucci, B.; Piquemal, J.-P. Towards Large Scale Hybrid QM/MM Dynamics of Complex Systems with Advanced Point Dipole Polarizable Embeddings. Chem. Sci. 2019, accepted for publication. http://dx.doi.org/10.1039/C9SC01745C.

(13) Lipparini, F.; Lagardère, L.; Raynaud, C.; Stamm, B.; Cancès, E.; Mennucci, B.; Schnieders, M.; Ren, P.; Maday, Y.; Piquemal, J. P. Polarizable molecular dynamics in a polarizable continuum solvent. J. Chem. Theory Comput. 2015, 11, 623-634.

(14) Wang, J.; Cieplak, P.; Li, J.; Wang, J.; Cai, Q.; Hsieh, M.; Lei, H.; Luo, R.; Duan, Y. Development of Polarizable Models for Molecular Mechanical Calculations II: Induced Dipole Models Significantly Improve Accuracy of Intermolecular Interaction Energies. J. Phys. Chem. B 2011, 115, 3100.

(15) Ponder, J. W.; Wu, C.; Ren, P.; Pande, V. S.; Chodera, J. D.; Schnieders, M. J.; Haque, I.; Mobley, D. L.; Lambrecht, D. S.; DiStasio Jr, R. A.; Head-Gordon, M.; Clark, G. N. I.; Johnson, M. E.; Head-Gordon, T. Current Status of the AMOEBA Polarizable Force Field. J. Phys. Chem. B 2010, 114, 2549-2564.

(16) Sala, J.; Guàrdia, E.; Masia, M. The polarizable point dipoles method with electrostatic damping: implementation on a model system. J. Chem. Phys. 2010, 133, 234101.

(17) Greengard, L.; Rokhlin, V. A fast algorithm for particle simulations. J. Comput. Phys. 1987, 73, 325-348.

(18) White, C. A.; Johnson, B. G.; Gill, P. M.; Head-Gordon, M. The continuous fast multipole method. Chem. Phys. Lett. 1994, 230, 8-16. 
(19) Beatson, R.; Greengard, L. A short course on fast multipole methods. Wavelets, Multilevel Methods and Elliptic PDEs. 1997; pp 1-37.

(20) Pérez-Jordá, J. M.; Yang, W. A concise redefinition of the solid spherical harmonics and its use in fast multipole methods. J. Chem. Phys. 1996, 104, 8003-8006.

(21) Frisch, M. J.; Trucks, G. W.; Schlegel, H. B.; Scuseria, G. E.; Robb, M. A.; Cheeseman, J. R.; Scalmani, G.; Barone, V.; Petersson, G. A.; Nakatsuji, H.; Li, X.; Caricato, M.; Marenich, A. V.; Bloino, J.; Janesko, B. G.; Gomperts, R.; Mennucci, B.; Hratchian, H. P.; Ortiz, J. V.; Izmaylov, A. F.; Sonnenberg, J. L.; WilliamsYoung, D.; Ding, F.; Lipparini, F.; Egidi, F.; Goings, J.; Peng, B.; Petrone, A.; Henderson, T.; Ranasinghe, D.; Zakrzewski, V. G.; Gao, J.; Rega, N.; Zheng, G.; Liang, W.; Hada, M.; Ehara, M.; Toyota, K.; Fukuda, R.; Hasegawa, J.; Ishida, M.; Nakajima, T.; Honda, Y.; Kitao, O.; Nakai, H.; Vreven, T.; Throssell, K.; Montgomery, J. A., Jr.; Peralta, J. E.; Ogliaro, F.; Bearpark, M. J.; Heyd, J. J.; Brothers, E. N.; Kudin, K. N.; Staroverov, V. N.; Keith, T. A.; Kobayashi, R.; Normand, J.; Raghavachari, K.; Rendell, A. P.; Burant, J. C.; Iyengar, S. S.; Tomasi, J.; Cossi, M.; Millam, J. M.; Klene, M.; Adamo, C.; Cammi, R.; Ochterski, J. W.; Martin, R. L.; Morokuma, K.; Farkas, O.; Foresman, J. B.; Fox, D. J. Gaussian Development Version Revision J.02. 2019; Gaussian Inc. Wallingford CT.

(22) Strain, M. C.; Scuseria, G. E.; Frisch, M. J. Achieving Linear Scaling for the Electronic Quantum Coulomb Problem. Science 1996, 271, 51-53.

(23) Caprasecca, S.; Jurinovich, S.; Lagardère, L.; Stamm, B.; Lipparini, F. Achieving Linear Scaling in Computational Cost for a Fully Polarizable MM/Continuum Embedding. J. Chem. Theory Comput. 2015, 11, 694-704.

(24) Rackers, J. A.; Wang, Z.; Lu, C.; Laury, M. L.; Lagardère, L.; Schnieders, M. J.; 
Piquemal, J.-P.; Ren, P.; Ponder, J. W. Tinker 8: Software Tools for Molecular Design. J. Chem. Theory Comput. 2018, 14, 5273-5289.

(25) Lagardère, L.; Jolly, L.-H.; Lipparini, F.; Aviat, F.; Stamm, B.; Jing, Z. F.; Harger, M.; Torabifard, H.; Cisneros, G. A.; Schnieders, M. J.; Gresh, N.; Maday, Y.; Ren, P. Y.; Ponder, J. W.; Piquemal, J.-P. Tinker-HP: a massively parallel molecular dynamics package for multiscale simulations of large complex systems with advanced point dipole polarizable force fields. Chem. Sci. 2018, 9, 956-972. 
Figure 4: Graphical Table of Contents

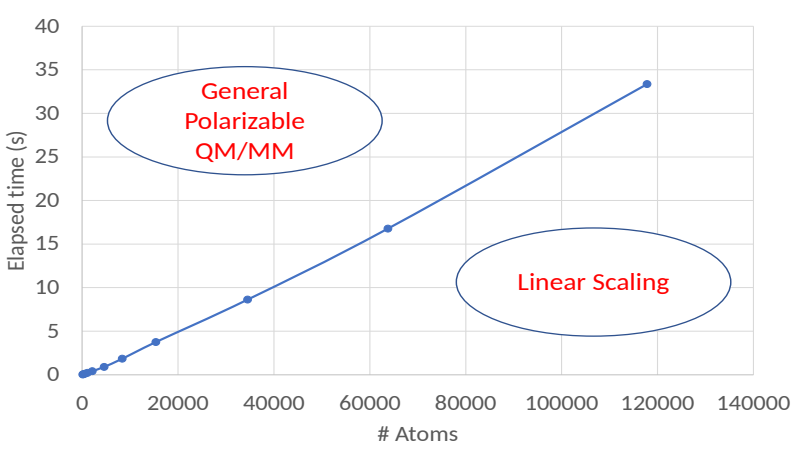

